\title{
Demostrar el impacto de los servicios: una prioridad de la Terapia Ocupacional
}

Alicia Trujillo Rojas, M.A., OTR*

\section{Introducción}

En Colombia es evidente el crecimiento de los servicios de terapia ocupacional en los campos de la salud, la educación, el trabajo, el bienestar social y la justicia. Sin embargo, aunque no existen estudios sobre número deseable de terapeutas ocupacionales por habitante, la cobertura es baja (1.01 terapeuta ocupacional por 10.000 habitantes) e inferior a la de otras profesiones como fisioterapia (1.49 fisioterapeutas por 10.000 habitantes) o fonoaudiología ( 1.75 fonoaudiólogos por 10.000 habitantes), según aproximaciones de la Superintendencia de Salud (1997) para el año 2000. Además, en lo tocante a eficacia e impacto, es frecuente el desconocimiento de diversos agentes sociales acerca de la especificidad y utilidad de la terapia ocupacional. Por otra parte, se ha venido reportando reducción de espacios laborales por motivos de reestructuración de organizaciones públicas y privadas, problemas financieros, interrogantes acerca del costo-beneficio de la atención y crisis generalizada del sector de los servicios. Estas realidades así mismo afectan a otras profesiones, debido a la situación económica, política y social que vive la nación. Sin embargo, se trata de hechos que deben ser cuidadosamente analizados al interior de la comunidad de terapeutas ocupacionales para determinar sus causas e introducir correctivos. De lo contrario, su ocurrencia continuada no favorece el impacto, o sea la demostración de las consecuencias prácticas favorables que ejerce la terapia ocupacional en las personas y la sociedad.

El mayor auge, al igual que la sostenibilidad de la profesión dependen de demostrar el cumplimiento de condiciones tales como: la especificidad de los servicios; el

\footnotetext{
* Profesora Titular Universidad Nacional de Colombia.
} 
costo-efectividad de los procedimientos y.tecnología que se aplican; y la utilidad real (impacto), demostrable en la mejor calidad de vida de las personas atendidas, como consecuencia de la atención de terapia ocupacional. Luego es fundamental el estudio y formulación de estrategias que contribuyan a conocer y controlar dichos factores, como propone este artículo.

Los objetivos que aquí se cumplen son: (a) reflexionar sobre la necesidad de asegurar un creciente impacto de los servicios de terapia ocupacional; $y$ (b) identificar $y$ comentar sobre algunos indicadores que permiten detectar, monitorear e incrementar el grado de impacto social de la terapia ocupacional.

\section{Posición teórica}

La conferencia sigue la siguiente línea de pensamiento: (a) el impacto social de una profesión está relacionado con factores tales cómo la calidad, la eficiencia, la eficacia y la cobertura, entre otros'; (b) los conceptos calidad, eficiencia, eficacia, cobertura y economía deben ser gerenciados, es decir medidos de manerá que su identificación haga posible la toma de decisiones conducente al direccionamiento más acorde con la misión, objetivos y metas institucionales y profesionales; $y$ (c) una gerencia que se guíe, entre otros, por criterios de calidad, eficiencia, eficacia y costo-efectividad es un mandato de la legislación vigente en seguridad social (Marquez, 2000, Rodríguez, 1999) y única manera de afrontar exitosamente las exigencias del mundo competitivo actual. Entiéndese la gerencia déla calidad como "el enfoque planeado y sistemático que se ocupa de diseñar, medir, evaluar y mejorar el desempeño organizacional durante todo el proceso de atención" (Forer, 1996, p.x). Un gerente efectivo es aquel que toma decisiones con base en un seguimiento permanente de todos los procesos de gestión organizacional.

\section{Marco legislativo}

La legislación más actual en el terreno de la gestión de la calidad se produce a partir de la Constitución Política de 1991. Surgen así leyes, decretos reglamentarios y normas que representan un mandato para el ejercicio de las profesiones y que

\footnotetext{
${ }^{1}$ Economía se refiere a la capacidad institucional para recibir, generar y movilizar productivamente los recursos financieros, de manera que contribuyan a cumplir su misión. Eficacia consiste en el grado de cumplimiento de los objetivos y metas planteados en términos de servicios profesionales a la pobla-
} 
abarcan los campos de la salud, la educación y el trabajo, en los cuales ejercen los terapeutas ocupacionales. El terapeuta ocupacional debe ser estricto y a la vez innovador en la interpretación y aplicación de la legislación. A continuación se identifica alguna de la normatividad más importante:

- Ley 100 de 1993, la cual se crea el Sistema de Seguridad Social Integral. Varios artículos de esta ley estipulan: el control y evaluación que se debe ejercer sobre la calidad (artículo 153-9, 227); el sistema de acreditación de las instituciones prestadoras de servicios (artículo 186); la veeduría comunitaria tendiente a garantizar la cobertura, eficiencia y calidad en la prestación de los servicios (artículo 231); y las estrategias para desarrollar requisitos esenciales, procesos de gestión efectivos y planes de mejoramiento de la calidad.

- Ley 87 de 1993 , a través de la cual se dictan normas para el ejercicio del control interno, entendido como la organización y conjunto de principios, planes, métodos, normas, procedimientos y mecanismos de verificación de todas las actividades institucionales.

- Ley 115 de 1994. o Ley General de Educación, la cual traza la estructura educativa más actualizada, de acuerdo con principios de servicios público, derechos ciudadanos y calidad. En el título III, capítulo I dicta normas sobre la integración de escolares con limitaciones, una póblación objeto de los programas de servicios de terapia ocupacional.

- Decreto 2082 de 1996 del Ministerio de Educación relacionado con disposiciones para asegurar la atención educativa de niños con limitaciones, de manera que tengan la oportunidad de alcanzar el desarrollo hümano y la integración social en las mejores circunstancias posibles.

- Decreto 2174 de 1996 del Ministerio de Salud, según el cual se organiza el sistema obligatorio de garantía de calidad del Sistema de Seguridad Social en Salud, el cual está a cargo de la Superintendencia Nacional de Salud y obliga a todos los prestadores de servicios, entre ellos los terapeutas ocupacionales.

- Resolución 3165 de 1996 del Ministerio de Salud: por la cual se adopta el manual de lineamientos de atención en salud para las personas con discapacidad. Esta norma representa un marco de referencia básico para la organiza-

ción objetivo. Eficiencia implica el cumplimiento de metas, con optimización de tiempós y recursos físicos y humanos. La calidad, asociada a la eficiencia y la eficacia, incluye la oportunidad, actualidad, suficiencia y seguridad de los servicios que se prestan. Cobertura es la magnitud en que un producto o servicio humano es disponible para todos los usuarios potenciales. 
ción y gestión de servicios de rehabilitación en todos los niveles de atención, terreno en el cual ejerce una proporción importante de terapeutas ocupacionales.

- Resolución 4252 de 1997 del Ministerio de Salud: por la cual se establecen las normas técnicas, científicas y administrativas sobre los requisitos esenciales para la prestación de servicios de salud y se publica el anexo técnico que identifica los requisitos esenciales. En esencia, esta norma contiene el instrumento a través del cual se operacionaliza el Sistema Obligatorio de Garantía de Calidad (Decreto 2174 de1996), el cual debe ser diligenciado regularmente por los prestadores de servicios, entre ellos el terapeuta ocupacional.

- Educación para todos, 1997: Lineamientos generales para la atención educativa a la población con limitaciones o capacidades excepcionales en los centros educativos del Distrito Capital. Con base en normas nacionales e internacionales, este documento determina la política tendiente a promover el desarrollo pleno de escolares con discapacidad.

- Resolución 1995 de 1999 del Ministerio de Salud: por la cual se establecen normas para el manejo de la historia clínica. Si bien esta norma enfatiza principalmente lo relacionado con la atención médica, es también de obligatorio cumplimiento para los terapeutas ocupacionales.

- Resolución 365 de 1999 del Ministerio de Salud: por la cual se adopta la clasificación única de procedimientos en el campo de la salud. Todos los prestadores de servicios, entre ellos los terapeutas ocupacionales, deben prestar y facturar servicios de acuerdo con esta norma.

- Resolución 2333 de 2000 del Ministerio de Salud, por la cual se modifica la Clasificación Unica de Procedimientos en Salud. Algunos de estos cambios contribuyen a dar cuenta más precisa de la especificidad de los procedimientos de terapia ocupacional.

- Norma Técnica Colombiana NTC-ISO 9001:2000. Esta norma del Instituto Colombiano de Normas Técnicas y Certificación ICONTEC, traducción de la norma internacional, orienta a las organizaciones sobre los requisitos que deben cumplir para la gestión y el aseguramiento de la calidad. Se conoce una investigación en curso, de estudiantes de terapia ocupacional de la Universidad Nacional de Colombia (Sanabria y Cáceres, 2002), que estudia la planeación de un servicio de terapia ocupacional bajo los criterios de gestión de la norma ISO 9001: 2000. 
En síntesis, la normatividad mencionada establece los derechos de los ciudadanos a la salud, la educación y el trabajo, regula la función social que deben cumplir los servicios, obliga a la participación ciudadana en órganos de dirección y control de las instituciones de servicios, resalta el derecho a la libre elección de empresas prestadoras de servicios y promueve el desarrollo de una cultura de la calidad. Esta orientación exige de las instituciones y proveedores de servicios un acelerado proceso de modernización, el cual requiere plantear y alcanzar metas relacionadas con: el seguimiento y mejoramiento de la calidad, la eficiencia y la competitividad; la introducción de mecanismos de control de la gestión; el diseño de medidas de control interno y auditoría; la medición de la satisfacción de usuarios; y el desarrollo de sistemas de información (Márquez, 2000). Al estar la comunidad de terapia ocupacional inmersa en el contexto de los servicios humanos, debe hacer propia la cultura del seguimiento, autoevaluación, evaluación externa y mejoramiento de todos sus programas y proyectos y entender que de ello depende su grado de permanencia social.

\section{Indicadores de Gerencia}

El seguimiento de los conceptos de calidad, eficiencia, eficacia y cobertura, esenciales para lograr impacto social, usualmente se efectúa a través de indicadores de gestión especialmente diseñados para representar esos conceptos. Algunos ejemplos de indicadores que contribuyen al monitoreo cuantitativo y cualitativo y que aquí se estiman cruciales para terapia ocupacional, son:

- Generación de recursos propios. La capacidad de los servicios de producir recursos en beneficio de la organización es un imperativo del momento actual porque, con frecuencia, de ello depende la permanencia de los programas.

- Inversión en tecnología de rehabilitación. El éxito de las metas de autonomía para los usuarios está asociada en alto grado con las ayudas tecnológicas a las que se tenga acceso.

- Grado en que la tecnología es actualizada, suficiente y segura. Un servicio de terapia ocupacional de calidad debe mantenerse al día en los avances científico tecnológicos que usualmente implican la aparición de instrumentos y artefactos más sofisticados, funcionales y estéticos o de mayor impacto.

- Grado en que el recurso humano tiene capacitación postgraduada y beneficio específico que aporta. Algunos países, entre ellos Estados Unidos y Canadá, 
empiezan a exigir el nivel educativo de maestría para el ejercicio de la terapia ocupacional, debido a la complejidad de los problemas que deben resolver estos profesionales.

- Porcentaje de terapeutas ocupacionales con cargos de visibilidad regional, nacional e internacional. Este indicador sugiere el grado de reconocimiento social de una profesión.

- Índice de desempleo. Habla de la demanda social de terapeutas ocupacionales, útil para tomar múltiples medidas educativas y de mercadeo.

- Índice de demanda y oferta de servicios de servicios por áreas y por poblaciones. Permite planear estrategias, a partir del reconocimiento de necesidades específicas del país y sus regiones.

- Vigencia de estándares profesionales y administrativos. Demuestra el grado en que las profesiones están en capacidad de autoregularse, de manera responsable.

- Grado de cumplimiento de estándares profesionales. Este es un indicador más evolucionado que el anterior, por cuanto se refiere a la aplicación de las normas establecidas, por el mayor número posible de miembros de la comunidad profesional.

- Tipo y costo de procedimientos aplicados según diagnóstico. A mayor evolución de las profesiones, mayor claridad y precisión sobre los procedimientos que emplea y su especificidad según entidades diagnósticas.

- Efectividad de procedimientos según diagnóstico. La práctica basada en la evidencia científica, de gran actualidad internacional, hace necesario imperioso demostrar de manera rigurosa la utilidad de los procedimientos que se emplean en beneficio de los usuarios.

- Diferencia entre los estados de admisión y salida de usuarios. Este indicador permite observar la efectividad de un programa o procedimientos aplicados.

- Proporción de metas cumplidas por metas planteadas. En el ejercicio contemporáneo es indispensable precisar el grado en que se cumple lo planeado, con miras a ajustes o reformulación de los planteamientos.

- Tiempo promedio para el cumplimiento de metas. Las exigencias actuales de calidad y rendimiento significan que el tiempo para cumplir el tratamiento o programa previsto debe ser cuidadosamente monitoreado.

- Proporción de usuarios internos y externos satisfechos. La opinión que sobre la terapia ocupacional tengan los pacientes, sus familias y otros profesionales y funcionarios relacionados es una muestra potente de la calidad del servicio. 
- Variación en la calidad de vida como consecuencia de los programas de terapia ocupacional. Este es un potente indicador de impacto, en cuanto muestra los efectos definitivos producto de los programas ofrecidos.

El comportamiento de los indicadores mencionados debe (a) registrarse de manera sistemática, para todos los profesionales y situaciones del servicio de terapia ocupacional; (b) consolidarse con una periodicidad determinada; $(c)$ integrarse al sistema de información de la organización; $y$ (d) emplearse con miras a fortalecer el proceso de planeación, evaluación y mejoramiento de los servicios.

\section{Productos esperados}

¿Cuáles son los beneficios que se pueden obtener a través de la práctica de construir y utilizar indicadores de gestión? La formulación y aplicación sistemática de indicadores es de gran utilidad para construir bases de datos y sistemas de información institucionales y de asociaciones gremiales como la Asociación Colombiana de Terapia Ocupacional, que permitan tomar decisiones inteligentes, entre otros, sobre:

- Diseño de estándares profesionales y administrativos válidos.

- Orientación de planes estratégicos.

- Precisión y operacionalización de criterios de autoevaluación, auditoría y acreditación de prógramas de atención.

- Direccionamiento de la demanda y oferta de servicios.

- Control de la eficacia y la eficiencia de la atención.

- Estrategias de conservación, reorientación y ampliación de campos profesionales.

En definitiva, el redireccionamiento que se viene presentando en la orientación y prestación de los servicios humanos en Colombia y en otros países de la región plantea retos de gran complejidad que los terapeutas ocupacionales deben asumir con una creciente sofisticación de las concepciones, las estrategias y los medios de gestión a su disposición. La gerencia eficiente de los servicios debe ocupar un espacio complementario e igualmente importante al que se da a a la formación científico-tecnológica. Esta complementariedad será fundamental para situar a la terapia ocupacional en lugares de creciente efectividad e impacto social. 


\section{Lista de referencias}

Forer, S. (1996). Outcome management and program évaluation made easy: a toolkit for occupational therapy practicioners, Bethesda, $\mathrm{MD}$ : American Occupational Therapy Association.

ICONTEC (2000) Norma técnica colombiana NTC-ISO 9001 Sistemas de gestión de calidad Requisitos. Bógotá: Autor.

Márquez; M. (2000): Reflexiones para terapia ocupacional sobre los sistemá de garan: tía de calidad y auditoría en salud Texto de una conferencia. Carrerarde Terapia Ocupacional, Universidad Nacional de Colombia.

Rodríguez, C.E. (1999). Calidad de atención en salud. Aspectôs jurídiços pollitićcos y técnicos desde el área de prestación de servicios. Documento. Ministerio de Salud.

Superintendencia Nacional de Salud (1997). El tálento humano de la salud en Co - Sombia. Santa Fe de Bocotá: Ministerio de Salud.

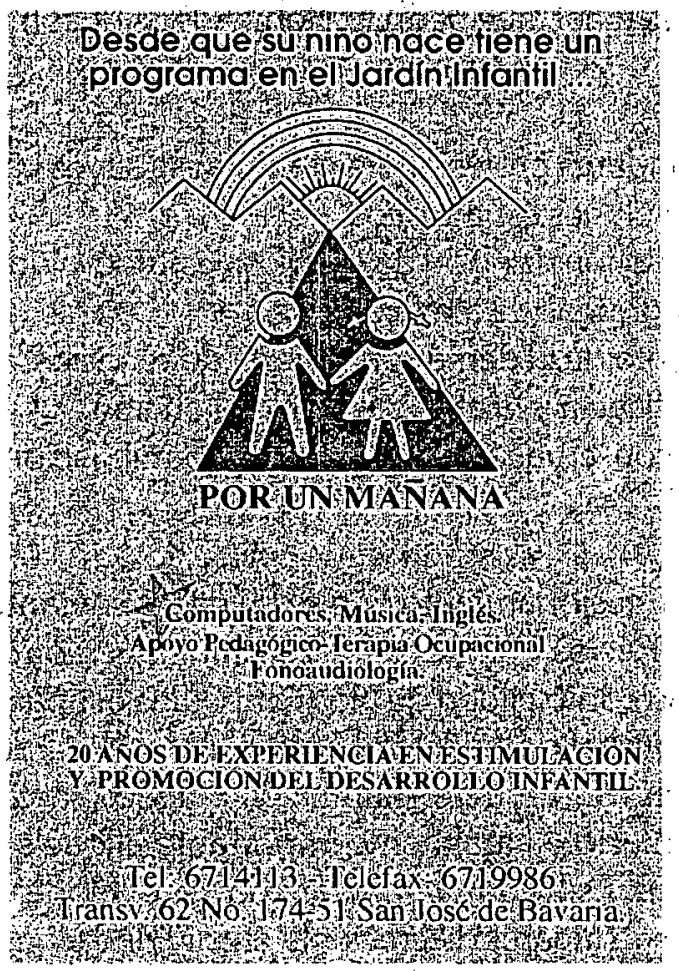

